\title{
Atom mirror etched from a hard drive
}

\author{
Benjamin Lev, ${ }^{\text {a) }}$ Yves Lassailly, ${ }^{\text {b) }}$ Chungsok Lee, Axel Scherer, and Hideo Mabuchi \\ California Institute of Technology, Pasadena, California 91125
}

(Received 1 April 2003; accepted 16 May 2003)

\begin{abstract}
We describe the fabrication of an atom mirror by etching of a common hard drive, and we report the observation of specular retroreflection of $11 \mu \mathrm{K}$ cesium atoms using this mirror. The atoms were trapped and cooled above the hard drive using the mirror magneto-optical trap technique, and upon release, two full bounces were detected. The hard drive atom mirror will be a useful tool for both atom optics and quantum computation. (C) 2003 American Institute of Physics.
\end{abstract}

[DOI: $10.1063 / 1.1592305$ ]

Laser cooling and trapping techniques have made possible the preparation of extremely cold samples of atoms. Atom optics employs elements such as mirrors, lenses, gratings, and beam splitters to manipulate these cold atoms in a fashion similar to the familiar photon optics. The advent of the Bose-Einstein condensation of neutral atoms has enhanced the importance of developing atom optical elements. In particular, atom mirrors-surfaces that reflect atomsplay a crucial role in the field of atom optics, and it is of keen interest to develop mirrors that are simple to fabricate yet highly specular. In this letter we demonstrate a straightforward technique to produce large area, high resolution permanent-magnetic structures on flat, rigid, and inexpensive substrates.

Several types of atom mirrors have been fabricated using evanescent light fields, ${ }^{1}$ dynamic magnetic fields, ${ }^{2}$ and static magnetic fields. ${ }^{3}$ Evanescent mirrors repulse atoms from a prism surface using a potential created by a blue-detuned light field. Although magnetic mirrors cannot generally be modulated as easily as evanescent mirrors, they do offer many advantages: passive operation, compactness (no laser access is needed), and much larger repulsive areas.

Magnetic mirrors employ a sheet of alternating current or magnetization to create an exponentially increasing potential near the mirror surface. ${ }^{4}$ To lowest order, this potential is proportional to $B_{0} e^{-k y}$. The surface field, $B_{0}$, sets the maximum atom energy that can be reflected, and the spatial period of the current or magnetization, $a=2 \pi / k$, determines the amount of time the atoms interact with the mirror. The magnetic mirror approximates a perfectly flat mirror as $B_{0}$ increases and $a$ decreases. For example, if $B_{0}=1 \mathrm{kG}$ and $a$ $=1 \mu \mathrm{m}$, a cesium atom in the $6^{2} S_{1 / 2} F=4, m_{F}=4$ state will be reflected when dropped from a height of $0.4 \mathrm{~m}$, and will only interact with the mirror for $5 \mu$ s if dropped from $2 \mathrm{~cm}$.

Mirrors made from serpentine patterns of wires can produce time-dependent reflection potentials. However, they have not been fabricated with periods smaller than $10 \mu \mathrm{m}$, and the power dissipated by the small wires requires cooling by liquid nitrogen and pulsed operation. ${ }^{5}$ Sinusoidal magnetization of audio-tape, floppy disks, and videotape can pro-

\footnotetext{
${ }^{a}$ Electronic mail: benlev@ caltech.edu

${ }^{b)}$ Present address: Laboratoire PHMC/Ecole Polytechnique, 91128 Palaiseau cedex, France.
}

duce magnetic mirrors with magnetization periods down to $12 \mu \mathrm{m} .{ }^{3}$ Mirrors made from millimeter-sized arrays of permanent magnets have been demonstrated, as have mirrors produced by $1-4 \mu \mathrm{m}$ periodic structures fabricated by sputtering ferromagnetic material onto a grooved substrate patterned by electron-beam lithography. ${ }^{6}$

We recently fabricated a magnetic mirror by etching a common hard drive, and we have used this mirror to retroreflect a cold cloud of $10^{6}$ cesium atoms. Hard drives offer several advantages for making and using atom mirrors. The common hard drive provides a large surface area of thin magnetic film whose surface is specifically designed to be very flat, smooth, and rigid. Furthermore, the film's remnant magnetic field and coercivity can be as large as 7 and $3 \mathrm{kG}$, respectively. ${ }^{7}$ An atom mirror could in principle be fabricated with a $2 \mu \mathrm{m}$ periodicity over the entire surface of the hard drive. Old or discarded hard drives may be used: an Apple hard drive from the mid-1990's was used for the experiment presented here.

We fabricate the mirror by etching $2 \mu \mathrm{m}$ wide, $\sim 100 \mathrm{~nm}$ deep trenches into a $1 \mathrm{~cm}^{2}$ section of the surface of the hard drive. These $100 \mathrm{~nm}$ trenches extend past the magnetic layer to form a periodic array of $1 \mu \mathrm{m}$ wide, $30 \mathrm{~nm}$ thick, and $1 \mathrm{~cm}$ long stripes of cobalt alloy (see Fig. 1). The cobalt alloy is granular, which enhances the coercivity and allows us to magnetize the material in plane and parallel to the short axis of the magnetic strips. The typical grain size is 20-50 nm, ${ }^{7}$ and we expect the magnetization to be uniform for our much larger features. We do not know the exact materials and thicknesses of the layers of the proprietary hard drive. However, etching $\sim 100 \mathrm{~nm}$ is sufficient to remove the magnetic layer.

Standard photolithography is used to create the etch

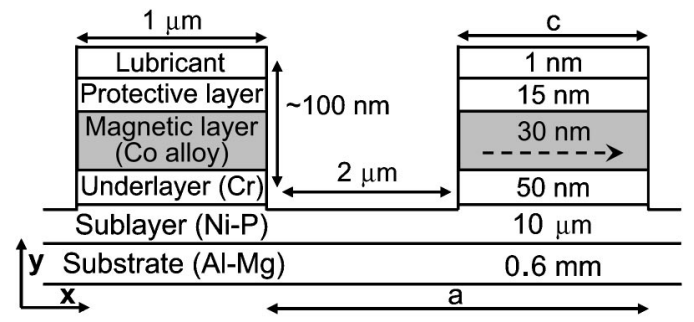

FIG. 1. A cross section of the etched hard drive. The magnetization is in-plane. See Ref. 7 for a description of the hard drive layers. 


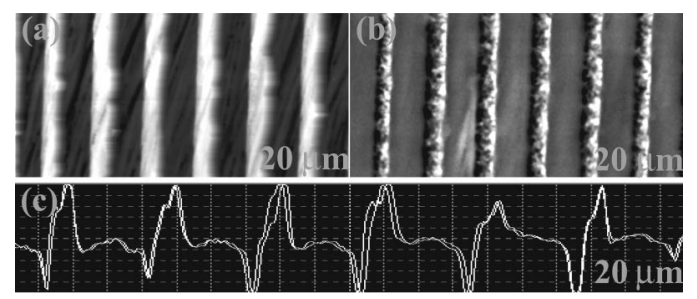

FIG. 2. $20 \mu \mathrm{m}$ wide (a) AFM scan, (b) MFM scan, and (c) MFM crosssection of the etched hard drive surface.

mask. After cutting the hard drive into $2-3 \mathrm{~cm}^{2}$ sections, positive photoresist (TSMR-8900, Tokyo Ohka Kogyo Co.) is spun onto the cleaned hard drive surface for $40 \mathrm{~s}$ at 4200 rpm. A $15 \mathrm{~s}$ exposure followed by $65 \mathrm{~s}$ in the developer (NMD-W 2.38\%) maps the photomask lines into the resist. The sample is ion milled with argon in a inductively coupled plasma (ICP) system. We etch for 8 minutes at a forward power of $100 \mathrm{~W}$, ICP power of $400 \mathrm{~W}$, and an argon flow of $40 \mathrm{sccm}$. The remaining photoresist is removed with acetone and, if necessary, a soft swab. To erase the hard drive's bits and magnetize it as a mirror, we insert the hard drive section into the field of an $8 \mathrm{kG}$ electromagnet whose field is parallel to the surface and perpendicular to the magnetic stripes.

The magnetic field from the etched hard drive, with inplane magnetization, $M_{0}$, parallel to the short axis of the magnetic stipes, is analogous to a periodic sheet of alternating in-plane magnetization $+M_{0} / 2$ and $-M_{0} / 2$. In the infinite array limit, the magnetic field above the surface is

$$
B^{2}=B_{1}^{2} e^{-2 k y}+2 B_{1} B_{3} \cos (2 k x) e^{-4 k y}+B_{3}^{2} e^{-9 k y}+\ldots,
$$

where $B_{1}=\mu_{0} M_{0}\left(1-e^{-k b}\right) / \pi, B_{3}=\mu_{0} M_{0}\left(1-e^{-3 k b}\right) / 3 \pi$, and $b=30 \mathrm{~nm}$ is the thickness of the magnetic layer. The field has no components in the $z$ direction, and rotates with a period equal to $a$ in the $x-y$ plane. Cesium atoms in the $F$ $=4, m_{F}=4$ state, which has the largest weak-field seeking magnetic moment, would have to be dropped from a height of $25 \mathrm{~cm}$ to penetrate to a height at which the second term in the expansion is equal the first, so to a good approximation the field may be written as

$$
B \approx B_{1} e^{-k y}+B_{3} e^{-3 k y} \cos (2 k x) .
$$

For our hard drive mirror, $B_{1}$ is equal to $2-4 \mathrm{kG}$ depending on the specific cobalt alloy. When $a=1 \mu \mathrm{m}$, the ratio of the first harmonic term to the purely exponential term for a cesium atom dropped from a height of $2 \mathrm{~mm}(20 \mathrm{~mm})$ is 1 $\times 10^{-6}\left(1 \times 10^{-3}\right)$ at the turning point $y=0.8 \mu \mathrm{m} \quad(y$ $=0.4 \mu \mathrm{m})$.

The etched hard drive used for the experiment has $a$ $\approx 3 \mu \mathrm{m}$ and $c \approx 1 \mu \mathrm{m}$ resulting in a ratio of magnetic layer to gap that is approximately 1:2. Figures 2(a) and 2(b) show $20 \mu \mathrm{m}$ wide atomic force microscopy (AFM) and MFM scans of the hard drive surface. The trenches in the AFM scan are dark, and the light to dark variation of magnetic strips shows the north and south poles of the magnetization. Figure 2(c) shows a $20 \mu \mathrm{m}$ cross section of the MFM scan: peaks represent the north and south poles. To describe the field above our etched hard drive, Eq. (2) can be modified to account for the deviation from a 1:1 width ratio by multiply- ing $B_{1}$ by $\sin (\pi c / a)$ and $B_{3}$ by $\sin (3 \pi c / a)$. In our device, the ratio of $c / a \approx 1 / 3$ decreases the $B_{1}$ term by 0.9 , but causes the corrugation term to nearly vanish.

The atom mirror is placed facing upwards in a vacuum chamber pumped to $5 \times 10^{-9}$ Torr. In contrast to the experiments that use a standard magneto-optical trap (MOT) to trap and cool the atoms $\sim 2 \mathrm{~cm}$ above the mirror, we use the mirror MOT technique to collect the atoms $1.5-4 \mathrm{~mm}$ above the surface. ${ }^{8}$ A MOT requires the zero of a magnetic quadrupole field to be centered at the intersection of six circularly polarized laser beams coming from all cardinal directions. To satisfy this configuration near the hard drive surface, two 1 $\mathrm{cm}$ diameter beams of opposite circular polarization reflect at $45^{\circ}$ from the $1 \mathrm{~cm}^{2}$ etched region. A retroreflected beam is positioned perpendicular to the $45^{\circ}$ beams and grazes the surface of the hard drive. Aligning the axis of the quadrupole field with one of the $45^{\circ}$ beams completes the mirror MOT configuration. The trapping lasers, each with an intensity of $4 \mathrm{~mW} / \mathrm{cm}^{2}$ and $1 \mathrm{~cm}$ wide, are detuned by $10 \mathrm{MHz}$ from cesium's $F=4, F^{\prime}=5$ cycling transition. A repumping beam tuned to the $F=3, F^{\prime}=4$ transition is superimposed onto both the grazing beam and a $45^{\circ}$ beam. The atoms are loaded from a thermal vapor.

In previous experiments using a perfectly reflecting gold mirror, we have been able to trap $2 \times 10^{6}$ cesium atoms in a mirror MOT and cool them to $3 \mu \mathrm{K}$. One might expect trapping and cooling to be much less effective with the etched hard drive due to its poor qualities as an optical mirror: the reflectivity is only $\sim 50 \%$, it is a good optical grating, and the magneto-optical Kerr effect degrades the circularity of the reflected $45^{\circ}$ beams. Nevertheless, we have been able to collect $1 \times 10^{6}$ atoms and subdoppler cool them to $11 \mu \mathrm{K}$. Achieving this low temperature is crucial because the atoms released directly from the mirror MOT, at a temperature of $\sim 120 \mu \mathrm{K}$, expand too quickly and become too diffuse to detect by the time they reach the hard drive surface.

The poor optical reflectivity of the mirror does slightly complicate the subdoppler cooling procedure; however, with careful zeroing of the magnetic field it is still possible to achieve polarization-gradient cooling to $11 \mu \mathrm{K}$ in a (downwards) moving reference frame. The atoms are optically pumped into the $F=4, m_{F}=4$ Zeeman substate just before being dropped, and we apply a $100 \mathrm{mG}$ bias field parallel to the magnetic stripes in order to maintain alignment of the atomic spins while they are falling/bouncing.

We have been able to detect two full bounces of the atoms from the hard drive atom mirror. Figures 3(a) and 3(b) show data from five runs of the experiment. The top panel shows the mean position of the atoms above the hard drive surface as a function of time. Superimposed is a curve depicting the expected trajectory of a particle falling under gravity and bouncing from a hard wall. The slope of a line fit to the lateral expansion of the falling atom cloud provides a measure of the atoms' rms velocity. A nonspecular mirror would heat and diffusely scatter the reflected atoms as they bounce, resulting in a sharp increase of the cloud expansion rate. We made a linear fit to prereflection $(t<15 \mathrm{~ms})$ data in each of the data sets, and deviation from this line, postreflection, would be evidence of nonspecularly. The dashed segment demarcates the region of unfitted data, and we do not AIP license or copyright, see http://apl.aip.org/apl/copyright.jsp 

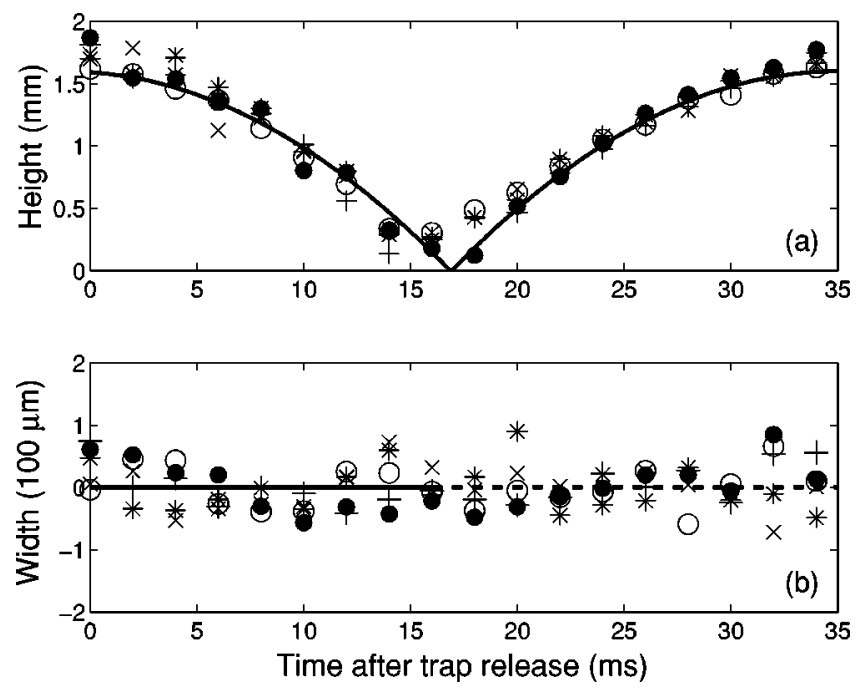

FIG. 3. Panel (a) shows the mean height of the atoms above the hard drive surface during the first bounce. Panel (b) shows the residuals from a linear fit to the width of the expanding atom cloud.

see any increase or offset of the residuals in this postreflection region: to within the experimental resolution, we do not detect any deviation from specular reflection.

We have realized a specular atom mirror built by etching a common hard drive. Magnetization periodicity of $3 \mu \mathrm{m}$ has been achieved, and we believe it would be straightforward to reduce this to $2 \mu \mathrm{m}$ with photolithography and to $\sim 1 \mu \mathrm{m}$ using a large area electron-beam writer. The hard drive atom mirror is compact, passive, relatively simple to fabricate, and possesses a large remanent magnetic field. Moreover, it has several desirable properties for applications beyond simple reflection of atoms. The hard drive's large coercivity should allow one to use wires fabricated directly on its surface to augment the mirror's ability to manipulate atoms. Likewise, electric pads could be printed on the surface. These pads would allow state-independent forces to act in concert with the state-dependent forces from the mirror's magnetic field to perform quantum logic gates necessary for quantum computation. ${ }^{9}$ The mirror can trap cold atom gases in two dimensions, and can act as an adjustable grating when used in conjunction with a magnetic bias field. ${ }^{10,11}$ Large area mirrors can be fabricated, and it seems possible that these mirrors could be useful for guiding or confining cold neutrons. ${ }^{12}$ As hard drive platters are expected to have good surface flatness and substrate rigidity, it may be possible to create two-dimensional waveguides and other devices by holding an opposing pair of atom mirrors a few microns apart.

The authors thank the Roukes group and Marko Loncar. This work was supported by the Multidisciplinary University Research Initiative program under Grant No. DAAD19-001-0374.

${ }^{1}$ V. Savalli, D. Stevens, J. Estève, P. Featonby, V. Josse, N. Westbrook, C. Westbrook, and A. Aspect, Phys. Rev. Lett. 88, 250404 (2002).

${ }^{2}$ A. Arnold, C. MacCormick, and M. Boshier, Phys. Rev. A 65, 031601 (2002).

${ }^{3}$ E. Hinds and I. Hughes, J. Phys. D 32, R119 (1999).

${ }^{4}$ G. Opat, S. Wark, and A. Cimmino, Appl. Phys. B: Photophys. Laser Chem. 54, 396 (1992).

${ }^{5}$ K. Johnson, M. Drndic, J. Thywissen, G. Zabow, R. Westervelt, and M. Prentiss, Phys. Rev. Lett. 81, 1137 (1998).

${ }^{6}$ A. Sidorov, R. McLean, F. Scharnberg, D. Gough, T. Davis, B. Sexton, G. Opat, and P. Hannaford, Acta Phys. Pol. B 33, 2137 (2002).

${ }^{7}$ R. L. Comstock, Introduction to Magnetism and Magnetic Recording (Wiley, New York, 1999).

${ }^{8}$ J. Reichel, W. Hansel, and T. Hansch, Phys. Rev. Lett. 83, 3398 (1999).

${ }^{9}$ J. Schmiedmayer, R. Folman, and T. Calarco, J. Mod. Opt. 49, 1375 (2002).

${ }^{10}$ E. Hinds, M. Boshier, and I. Hughes, Phys. Rev. Lett. 80, 645 (1998).

${ }^{11}$ P. Rosenbusch, B. Hall, I. Hughes, C. Saba, and E. Hinds, Phys. Rev. A 61, 031404(R) (2000).

${ }^{12}$ V. Vladimirskî, Sov. Phys. JETP 12, 740 (1961). 